\title{
DIS Structure Functions in Lattice QCD ${ }^{*, \dagger}$
}

\author{
Stefano Capitani ${ }^{a, \ddagger}$ and Giancarlo Rossi ${ }^{\mathrm{b}}$ \\ ${ }^{a}$ University of Southampton, Dept. of Physics, Highfield, Southampton SO17 1BJ, United Kingdom \\ bDipartimento di Fisica, Università di Roma "Tor Vergata" and INFN, Sezione di Roma II, \\ Via della Ricerca Scientifica 1, I-00133 Roma - Italy
}

In this talk I present the complete 1-loop perturbative computation of the renormalization constants and mixing coefficients of quark and gluon lattice operators of rank two and three whose hadronic elements enter in the determination of the first and second moment of Deep Inelastic Scattering Structure Functions, making use of the nearest-neighbor improved "clover-leaf" lattice QCD action.

To perform the huge amount of calculations required for the evaluation of all the relevant Feynman diagrams, extensive use of symbolic manipulation languages like Schoonschip and Form has been made.

\section{Introduction}

The computation on the lattice of renormalization factors is a necessary ingredient to connect lattice operators and matrix elements to their continuum counterparts and extract physical quantities from Monte Carlo results. We report here on the complete computation of the renormalization constants and mixing coefficients of the quark and gluon operators of rank two and of the quark operators of rank three [1] that measure the first two moments of Deep Inelastic Scattering Structure Functions (SF's). We have computed all these constants using the Sheikholeslami-Wohlert (SW) $O(a)$ improved action with $c_{S W}=1$ in 1-loop perturbation theory. Improvement 24] reduces the systematic error associated with the finiteness of the lattice spacing $a$, which is lowered, for on-shell quantities, from $O(a)$ to $O(a / \log a)$.

To work out the huge expressions arising for each of the numerous Feynman diagrams, it has been necessary to use extensively the algebraic computer manipulation languages Schoonschip and Form.

\footnotetext{
*Work supported in part by the EC Contracts "Computational Particle Physics" ERBCHBGCT940665 and ERBCHRXCT920051.

${ }^{\dagger}$ Presented by Stefano Capitani.

${ }^{\ddagger}$ From October 1996 at the Desy Theory Group.
}

\section{Moments of Structure Functions}

The hadronic tensor $W_{\mu \nu}$, from which the SF's are defined, is given in terms of the hadronic currents by

$W_{\mu \nu}=\frac{1}{2 \pi} \int d^{4} x e^{i q x}\left\langle p\left|J_{\mu}(x) J_{\nu}(0)\right| p\right\rangle$.

Using the Wilson OPE near the light-like region, $A(x) B(0) \sim \sum_{N, i} c_{N, i}\left(x^{2}\right) x^{\mu_{1}} \cdots x^{\mu_{N}} O_{\mu_{1} \cdots \mu_{N}}^{(N, i)}(0)$, the local product of operators can be expressed in terms of a set of symmetric and traceless operators $O_{\mu_{1} \cdots \mu_{N}}^{(N, i)}$ with vanishing vacuum expectation values. The matrix elements of the dominant (twist two) operators in the expansion, $O_{\mu_{1} \cdots \mu_{N}}^{(N)}$, have the general form

$\left\langle p\left|O_{\mu_{1} \cdots \mu_{N}}^{(N)}\right| p\right\rangle=A_{N}(\mu) p_{\mu_{1}} \cdots p_{\mu_{N}}+$ traces.

The formula for the moments of the SF's becomes

$\left\langle\mathrm{x}^{N-1}\right\rangle=\int d \mathrm{xx}^{N-1} \mathcal{F}_{k}\left(q^{2}, \mathrm{x}\right)=C_{N}\left(\frac{q^{2}}{\mu^{2}}\right) A_{N}(\mu),(3)$

where $\mathcal{F}_{1}=2 F_{1}, \mathcal{F}_{2}=F_{2} / \mathrm{x}$ and $\mathcal{F}_{3}=F_{3}$. The coefficients $C_{N}$ are known from continuum perturbation theory. From (3) we can extract $\left\langle\mathrm{x}^{N-1}\right\rangle$ knowing the corresponding matrix element $\left\langle p\left|O_{\mu_{1} \cdots \mu_{N}}^{(N)}\right| p\right\rangle$. The latter contains long distance (non-perturbative) physics, thus the only viable way to compute the moments of SF's is with the use of lattice methods. 
We have considered in our calculations the unpolarized SF's, and in particular we have computed the renormalization constants and mixing coefficients of the operators

$$
\begin{aligned}
& O_{\mu \nu}^{q}=\frac{1}{4} \bar{\psi} \gamma_{\{\mu} \overleftrightarrow{D}_{\nu\}} \psi \quad \longrightarrow \quad\langle\mathrm{x}\rangle_{q} \\
& O_{\mu \nu}^{g}=\sum_{\rho} \operatorname{Tr}\left[F_{\{\mu \rho} F_{\rho \nu\}}\right] \quad \longrightarrow \quad\langle\mathrm{x}\rangle_{g} \\
& O_{\mu \nu \tau}^{q}=\frac{1}{8} \bar{\psi} \gamma_{\{\mu} \overleftrightarrow{D}_{\nu} \overleftrightarrow{D}_{\tau\}} \psi \quad \longrightarrow \quad\left\langle\mathrm{x}^{2}\right\rangle_{q}
\end{aligned}
$$

where $D_{\mu}$ is the covariant derivative.

\section{Sheikholeslami-Wohlert improvement}

The matrix elements appearing in (2) need the computation of two- and three-point correlation functions [5]. To reduce discretization errors the SW improved action is used [4]. Adding to the Wilson action the "clover-leaf" SW term

$\Delta S_{I}^{f}=-i g_{0} a^{4} \sum_{n, \mu \nu} \frac{r}{4 a} \bar{\psi}_{n} \sigma_{\mu \nu} F_{n, \mu \nu} \psi_{n}$

and performing on the spinor fields in the operators $O_{\mu \nu}^{q}$ and $O_{\mu \nu \tau}^{q}$ the "rotations"

$\psi \longrightarrow\left(1-\frac{a r}{2} \overrightarrow{\not D}\right) \psi, \bar{\psi} \longrightarrow \bar{\psi}\left(1+\frac{a r}{2} \overleftarrow{\not D}\right)$

leads, in on-shell matrix elements [3], to a cancellation of all terms that in the continuum limit $\left(g_{0}^{2} \sim 1 / \log a\right)$ are effectively of order " $a$ " [4. 6 .

The systematic error related to the lattice discretization drops in this way from order $a$ to order $a / \log a$. Numerically this results in a substantial improvement. In fact, while the magnitude of the order $a$ terms is about 20-30\%, the magnitude of the order $a / \log a$ terms turns out to be about 5-10\%.

\section{Renormalization constants}

The renormalization constants connect the bare lattice operators, $O(a)$, to finite operators, $\widehat{O}(\mu)$, renormalized at a scale $\mu$ :

$\widehat{O}^{l}(\mu)=Z_{l k}(\mu a) O^{k}(a)$.

In the flavor Singlet case there is a mixing between quark and gluon operators of the same rank that have the same conserved quantum numbers. We then write:

$$
\begin{aligned}
& \widehat{O}^{q}=Z_{q q} O^{q}+Z_{q g} O^{g} \\
& \widehat{O}^{g}=Z_{g q} O^{q}+Z_{g g} O^{g},
\end{aligned}
$$

and in this case all elements of the mixing matrix

$$
\left(\begin{array}{cc}
\left\langle q\left|O^{q}\right| q\right\rangle & \left\langle g, \sigma\left|O^{q}\right| g, \sigma\right\rangle \\
\left\langle q\left|O^{g}\right| q\right\rangle & \left\langle g, \sigma\left|O^{g}\right| g, \sigma\right\rangle
\end{array}\right)
$$

have to be computed.

To this mixing (already present in continuum QCD) the lattice regularization adds additional mixings, induced by the breaking of (Euclidean) Lorentz invariance [7]. It is possible, by a careful choice of the Lorentz indices, to simplify at least partially the lattice mixing pattern. However, the higher the moment the more complicated the mixing pattern becomes [1,8]. In particular, the non-Singlet operators $O_{\{12\}}^{q}$ and $O_{\{123\}}^{q}$ are multiplicatively renormalizable on the lattice in the quenched approximation, but another useful second moment operator, $O_{\text {DIS }}^{q} \equiv O_{\{411\}}^{q}-$ $-\frac{1}{2}\left(O_{\{422\}}^{q}+O_{\{433\}}^{q}\right)$, is not.

The breaking of Lorentz invariance forced us to develop special computer routines to correctly perform the Dirac algebra on the lattice. They play a key role in our codes which are designed to automatically carry out all the steps of the necessary algebraic manipulations, starting from the elementary building blocks of each Feynman diagram.

\section{Some results}

Putting $Z=1+g_{0}^{2} / 16 \pi^{2}(\gamma \log a \mu+B)$, one gets for the $B$ 's and $\gamma$ 's of the first moment operators the results of Tables 1 and 2 . The numbers reported here for the $B$ 's are for $r=1$ and differ slightly from the ones published in the first paper of 11. They have been re-computed by us and cross-checked against the works in [9].

Simulations have been performed in the past with the unimproved Wilson action [5] 8 ]. Within errors, Monte Carlo results are consistent with experiment. No simulation has been as yet performed in the improved case. Values of the renormalization constants in the improved theory are somewhat larger than in the standard Wilson 


\begin{tabular}{|c|r|c|r|}
\hline \multicolumn{2}{|c|}{ Wilson } & \multicolumn{2}{c|}{ Improved } \\
\hline$B_{q q}^{W}$ & -3.165 & $B_{q q}^{I}$ & -15.816 \\
$B_{q g}^{W}$ & 0.208 & $B_{q g}^{I}$ & -0.743 \\
$B_{g q}^{W}$ & -5.817 & $B_{g q}^{I}$ & -4.044 \\
$\left(B_{g q}^{f}\right)^{W}$ & -2.168 & $\left(B_{q g}^{f}\right)^{I}$ & -6.084 \\
\hline \multicolumn{3}{|c|}{$B_{g g}=-15.585$} \\
\hline
\end{tabular}

Table 1

The lattice constants $B$ for the first moment; $B_{g g}^{f}$ corresponds to the quark loop contribution to the gluon propagator.

\begin{tabular}{|r|r|r||c|r|}
\hline \multirow{3}{*}{$B_{q q}$} & $\begin{array}{r}\text { sails } \\
\text { vertex }\end{array}$ & -4 & \multicolumn{2}{|c|}{} \\
& $5 / 9$ & \multicolumn{2}{|c|}{ ANOMALOUS } \\
& Delf-energy & -1 & \multicolumn{2}{c|}{ DIMENSIONS } \\
\cline { 2 - 5 } & total & $-34 / 9$ & $\gamma_{q q}$ & $16 / 3$ \\
\hline \multicolumn{2}{|c|}{$B_{q g}$} & $-4 / 9$ & $\gamma_{q g}$ & $4 / 3$ \\
\hline$B_{g q}$ & $-22 / 9$ & $\gamma_{g q}$ & $16 / 3$ \\
\hline$B_{g g}$ & $-4 / 3$ & $\gamma_{g g}$ & 0 \\
\hline$B_{g g}^{f}$ & $-10 / 9$ & $\gamma_{g g}^{f}$ & $4 / 3$ \\
\hline
\end{tabular}

Table 2

The continuum constants $B$ in the $\overline{M S}$ scheme and the anomalous dimensions for the first moment.

case. As an example, we give here a selection of the results for quark operators (for the complete results see [1]). At $\beta=6.0$ and for $r=1$ one gets 4

$$
\begin{aligned}
& \left(\widehat{O}_{\{12\}}^{q}\right)^{\mathrm{W}}=1.027\left(O_{\{12\}}^{q}\right)^{\mathrm{W}} \\
& \left(\widehat{O}_{\{12\}}^{q}\right)^{\mathrm{I}}=1.134\left(O_{\{12\}}^{q}\right)^{\mathrm{I}} \\
& \left(\widehat{O}_{\{123\}}^{q}\right)^{\mathrm{W}}=1.160\left(O_{\{123\}}^{q}\right)^{\mathrm{W}} \\
& \left(\widehat{O}_{\{123\}}^{q}\right)^{\mathrm{I}}=1.252\left(O_{\{123\}}^{q}\right)^{\mathrm{I}} \\
& \left(\widehat{O}_{\mathrm{DIS}}^{q}\right)^{\mathrm{W}}=\frac{1}{3}\left[1.184\left(O_{A}\right)^{\mathrm{W}}+1.156\left(O_{B}\right)^{\mathrm{W}}\right] \\
& \left(\widehat{O}_{\mathrm{DIS}}^{q}\right)^{\mathrm{I}}=\frac{1}{3}\left[1.331\left(O_{A}\right)^{\mathrm{I}}+1.187\left(O_{B}\right)^{\mathrm{I}}\right],
\end{aligned}
$$

where the first of each pair shows the Wilson results (for which we are in agreement with [8]) and the second the improved results.

Finally, we want to mention that results now exist also for the polarized SF's, though limited to the Wilson case [8].

\footnotetext{
${ }^{4}$ We have decomposed $O_{\mathrm{DIS}}^{q}=\frac{1}{3}\left(O_{A}+O_{B}\right)$ into the non-symmetric $O_{A} \equiv O_{411}^{q}-\frac{1}{2}\left(O_{422}^{q}+O_{433}^{q}\right)$ and $O_{B} \equiv O_{141}^{q}+O_{114}^{q}-\frac{1}{2}\left(O_{242}^{q}+O_{224}^{q}+O_{343}^{q}+O_{334}^{q}\right)$.
}

We thank C. McNeile, A. Pelissetto and H. Perlt for discussions and for correspondence.

\section{REFERENCES}

1. S. Capitani and G.C. Rossi, Nucl. Phys. B433 (1995) 351, and errata to be published; in Proceedings of the AIHENP95 International Workshop, eds. B. Denby and D. Perret-Gallix (World Scientific, Singapore, 1995); G. Beccarini, M. Bianchi, S. Capitani and G.C. Rossi, Nucl. Phys. B456 (1995) 271; S. Capitani, hep-ph 9607019, to be published in the Proceedings of the DIS96 International Workshop (World Scientific).

2. K. Symanzik, in Mathematical Problems in Theoretical Physics, ed. R. Schrader et al., Springer Lecture Notes in Physics, vol. 153 (1982) p. 47.

3. M. Lüscher and P. Weisz, Comm. Math. Phys. 97 (1985) 59.

4. B. Sheikholeslami and R. Wohlert, Nucl. Phys. B259 (1985) 572.

5. G. Martinelli and C.T. Sachrajda, Phys. Lett. B196 (1987) 184; Nucl. Phys. B306 (1988) 865; Nucl. Phys. B316 (1989) 355.

6. G. Heatlie, G. Martinelli, C. Pittori, G.C. Rossi and C.T. Sachrajda, Nucl. Phys. B352 (1991) 266 and Nucl. Phys. B (Proc. Suppl.) 17 (1990) 607; E. Gabrielli, G. Martinelli, C. Pittori, G. Heatlie and C.T. Sachrajda, Nucl. Phys. B362 (1991) 475; R. Frezzotti, E. Gabrielli, C. Pittori and G.C. Rossi, Nucl. Phys. B373 (1992) 781.

7. M. Baake, B. Gemünden and R. Oedingen, J. Math. Phys. 23 (1982) 944; J. Mandula, G. Zweig and J. Govaerts, Nucl. Phys. B228 (1983) 91 and 109.

8. M. Göckeler, R. Horsley, E.-M. Ilgenfritz, H. Perlt, P. Rakow, G. Schierholz and A. Schiller, Nucl. Phys. B (Proc. Suppl.) 42 (1995) 337; Phys. Rev. D53 (1996) 2317; hep-lat 9602029; hep-lat 9603006.

9. G. Burgio, S. Caracciolo and A. Pelissetto, hep-lat 9607010; C. McNeile, private communication. 